\title{
Use of NANA, a novel method of dietary assessment, for the longitudinal capture of dietary intake in older adults
}

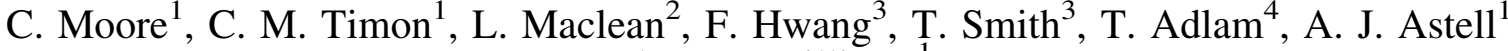 \\ and E. A. Williams \\ ${ }^{1}$ Human Nutrition Unit and ScHARR, Faculty of Medicine, Dentistry \& Health, University of Sheffield, Sheffield S10 2RX, \\ ${ }^{2}$ School of Psychology, St Andrews, Fife, KY16 9JP, ${ }^{3}$ School of Systems Engineering, University of Reading, Reading, RG6 \\ $6 A Y$ and ${ }^{4}$ BIME, Royal United Hospital, Bath, BAl $3 N G$, UK
}

Assessment of dietary intake can be particularly challenging in an older adult population. NANA is a touch-screen computer based dietary assessment tool that allows older adults to itemise and photograph the food and drink they are consuming for subsequent analysis by a nutritionist. Voice recordings are used to report food consumed outside the home and to report any items not found in the NANA database of foods. The NANA dietary assessment tool has been previously validated at a single collection point against a four-day estimated food diary. The aim of this study was to assess the feasibility of using the NANA system for the longitudinal capture of dietary intake data and to determine whether data quantity and quality are compromised with repeated use of the system.

Forty adults aged 65 years and older were recruited from Sheffield and St Andrews and asked to use the NANA system for 7 days on 3 occasions at the start of 3 consecutive months. The average age of the participants was 72 years and $60 \%$ were female. Four days of dietary intake data from each of the 3 NANA recording periods were analysed using WinDiets dietary analysis software (Robert Gordon University, Aberdeen, UK). The table shows the mean daily nutrient intake and the frequency of data entry across the 3 recording periods.

\begin{tabular}{|c|c|c|c|c|c|c|c|}
\hline & \multicolumn{2}{|c|}{ Period 1} & \multicolumn{2}{|c|}{ Period 2} & \multicolumn{2}{|c|}{ Period 3} & \multirow[b]{2}{*}{$P$} \\
\hline & Mean & SD & Mean & SD & Mean & SD & \\
\hline Energy (KJ/day) & 6487 & 1605.3 & 6732 & 1414.6 & 6309 & 1557.3 & 0.077 \\
\hline Fat (g/day) & 63.8 & 22.3 & 64.9 & 19.9 & 60.9 & 20.2 & 0.258 \\
\hline Carbohydrate (g/day) & 173 & 53.3 & 184 & 57.2 & 174 & 53.6 & 0.204 \\
\hline Protein (g/day) & 65.4 & 16.2 & 69.3 & 15.5 & 63.5 & 16.9 & 0.069 \\
\hline NSP (g/day) & 12.3 & 5.0 & 11.9 & 4.5 & 11.6 & 4.2 & 0.412 \\
\hline Vitamin C (mg/day) & 93.5 & 51.7 & 93.9 & 55.5 & 84.4 & 46.5 & 0.382 \\
\hline Calcium (mg/day) & 770 & 294.9 & 782 & 222.4 & 726 & 218.1 & 0.243 \\
\hline Iron (mg/day) & 10.5 & 4.2 & 10.1 & 3.0 & 9.9 & 2.9 & 0.531 \\
\hline Data entries/week & 41.3 & 18.45 & 42.4 & 18.06 & 38.7 & 15.12 & 0.075 \\
\hline Voice recordings/week & 6.0 & 4.49 & 5.5 & 4.23 & 5.2 & 4.84 & 0.097 \\
\hline
\end{tabular}

NSP: Non starch polysaccharides.

Significance tested using Repeated Measures Analysis of Variance.

There was no significant difference in mean daily nutrient intake, the number of data entries into the system or the number of voice recordings made across the 3 recording periods. The data demonstrates the consistency of nutrient intake in an older adult population over a 3 month period. The data also show that older adults were compliant with repeated data capture and consistent in their use of the NANA system, with no evidence of data collection fatigue. This study suggests that NANA is amenable to longitudinal data capture of dietary intake in older adults.

This work was funded by the ESRC New Dynamics of Ageing Programme. 